\title{
Anatomical and Functional Success Analysis of Lacrimal Canalicular Lacerations Repaired with Bicanalicular Stents in a Tertiary Referral Teaching Center in Mexico
}

\author{
Marisol Iñiguez-Soto ${ }^{1}$, Julián Espinosa-Noguera ${ }^{1}$, Adriana Dávila-Camargo ${ }^{1}$, Ángel Nava-Castañeda ${ }^{1}$ \\ ${ }^{1}$ Oculoplastics Department, Instituto de Oftalmología Conde de Valenciana, Chimalpopoca 14, Mexico City, Mexico
}

\begin{abstract}
Introduction: Functional and anatomical success after canalicular laceration repair using only Crawford bicanalicular stents was evaluated in a ophthalmological teaching center. The objective of this study was to evaluate functional and anatomical success after canalicular laceration repair using Crawford bicanalicular stents.
\end{abstract}

Materials and methods: Records of patients with canalicular laceration repair performed from 2010 to 2019 at Ophthalmology Institute Conde de Valenciana in Mexico City were reviewed. Demographic data, injury mechanism and complications were recorded. Anatomical success was assessed with canalicular irrigation and functional success was evaluated using Munk score. Phi correlation coefficient was used to compare the correlation between epiphora and lack of permeability of injured canaliculus and the presence of complications at 6 th month postoperative visit.

Results: Two-hundred eight patients with lacrimal canalicular injury reconstruction were documented during the study period. The most common age of presentation and etiology was from 21 to 30 years old and injury with a sharp object, respectively. 96 patients were included for the correlation analysis. At 6 th month, anatomical success was found in $75 \%$ and functional success was found in $77.8 \%$. A statistically significant and directly proportional linear was found between the presence of epiphora and lack of permeability of injured canaliculus, $(\mathrm{r} \varphi 0.76, \mathrm{p}<0.05)$ and between the presence of epiphora and postoperative complications, $(\mathrm{r} \varphi 0.509, \mathrm{p}<0.05)$.

Conclusion: Crawford bicanalicular stents are a valid and accessible option for canalicular laceration repair. Our success rate of lacrimal canalicular lacerations repaired with bicanalicular stents in a Mexican teaching hospital matches worldwide literature. Factors involved in the functional and anatomical success of the reconstruction include presence of postoperative complications and permeability of canaliculus after surgery.

Key words: Bicanalicular stents, Canalicular intubation, Canalicular laceration, Epiphora.

\begin{tabular}{lll}
\hline Financial Interest $:$ Nil & Received : 15.07.2020 \\
Conflict of Interest $:$ Nil & Accepted : 21.01.2021
\end{tabular}

Access this article online

Website: www.nepjol.info/index.php/NEPJOPH 


\section{INTRODUCTION}

Injury to the lacrimal canaliculus is a frequent complication of facial trauma involving the orbit (Wu et al, 2010). Etiology of canalicular lacerations varies depending on the country of the reviewed series and the age of patients; dog bites in children and assault or accidents in young adults (Jordan et al, 2008). Correct diagnosis and well-timed management is essential to prevent post-traumatic epiphora. Over the past 80 years, various surgical techniques, instruments and materials have been proposed for the reconstruction of canalicular lacerations (Reifler, 1991).

Silicon tubes are used as intubation stents to help safeguard the canaliculi function after healing and it helps surgical tissue relocation for a correct layered reconstruction (Liang et al, 2012). These intubation systems can be bicanalicular, like Crawford and Ritleng, or unicanalicular like Monoka stents (Sendul et al, 2015). Advantages and disadvantages exist for both intubation systems, and multiple reviews have covered this topic. Even though anatomical success can be achievable in most patients, postoperatively complaints of epiphora, blurred vision and foreign body sensation are described (Tint et al, 2011; Wladis et al, 2019).

Depending on the characteristics of the laceration, several controversies may exist regarding the surgical planning. For example, the importance of the superior versus the inferior canaliculus in tear drainage function and when damaged, if it should be repaired or not, as well as to repair one canaliculus when the other one is not involved or touch the uninvolved canaliculus in an attempt to repair the lacerated canaliculus (Reifler, 1991; Tint et al, 2011). Time for intervention has usually been established for 48 hours to improve the outcome, but reports of up to 10 days with successful results have been described (Naik et al, 2008).

This report presents the demographic and clinical characteristics, treatment outcomes and correlation between the presence of epiphora and the anatomical and functional success after 6 months of canalicular laceration repair in a tertiary teaching center using bicanalicular Crawford stents in Mexico City.

\section{MATERIALS AND METHODS}

An observational, retrospective, cross-sectional, and descriptive study was carried out at the Conde de Valenciana Ophthalmology Institute in Mexico City from 2010 to 2019. The study included patients who had suffered lacrimal canalicular. The international principles on investigation issued in the Declaration of Helsinki were respected. The studied variables were gender, age, injured side, involved canaliculus, time lapse between the injury and its repair, period of permanence of the silicone stent and complications. Follow-up was for at least 6 months. Data was retrieved from the electronic clinical records of the institute. The study was approved by the Ethics and Research Committee of the Conde de Valenciana Ophthalmology Institute (CC-013-1015). 
Canalicular laceration was diagnosed by identifying an irrigation probe at the proximal lumen of the canalicular tear. Most procedures were performed by an oculoplastic fellow under direct supervision of an oculoplastic surgeon attending. All procedures were performed under general anesthesia with Crawford bicanalicular stents (Eagle Labs, USA). The injured canaliculus and distal lumen were identified with surgical loupes and careful dissection with cotton swabs was carried out to identify the lacerated lumen. Nose was prepped with oxymetazoline $0.05 \%$ (Afrin, Bayer) and nasal packing. The stent was recovered from the nose with a blunt grooved probe. We prefer this maneuver over the Crawford retrieving hook because we consider it less traumatizing for the nasal mucosa. The stent in position allows us a posterior and medial traction to adequately suture in the correct plane of the eyelid, starting with the lacerated canaliculi, with the technique described by Kersten and Kulwin (Kersten et al, 1996). A polyglactin 6-0 suture (Coated Vycryl 910, Ethicon, USA) was used for canaliculus and injured eyelid structures repair (fig. 1). Following the surgery, each patient was treated
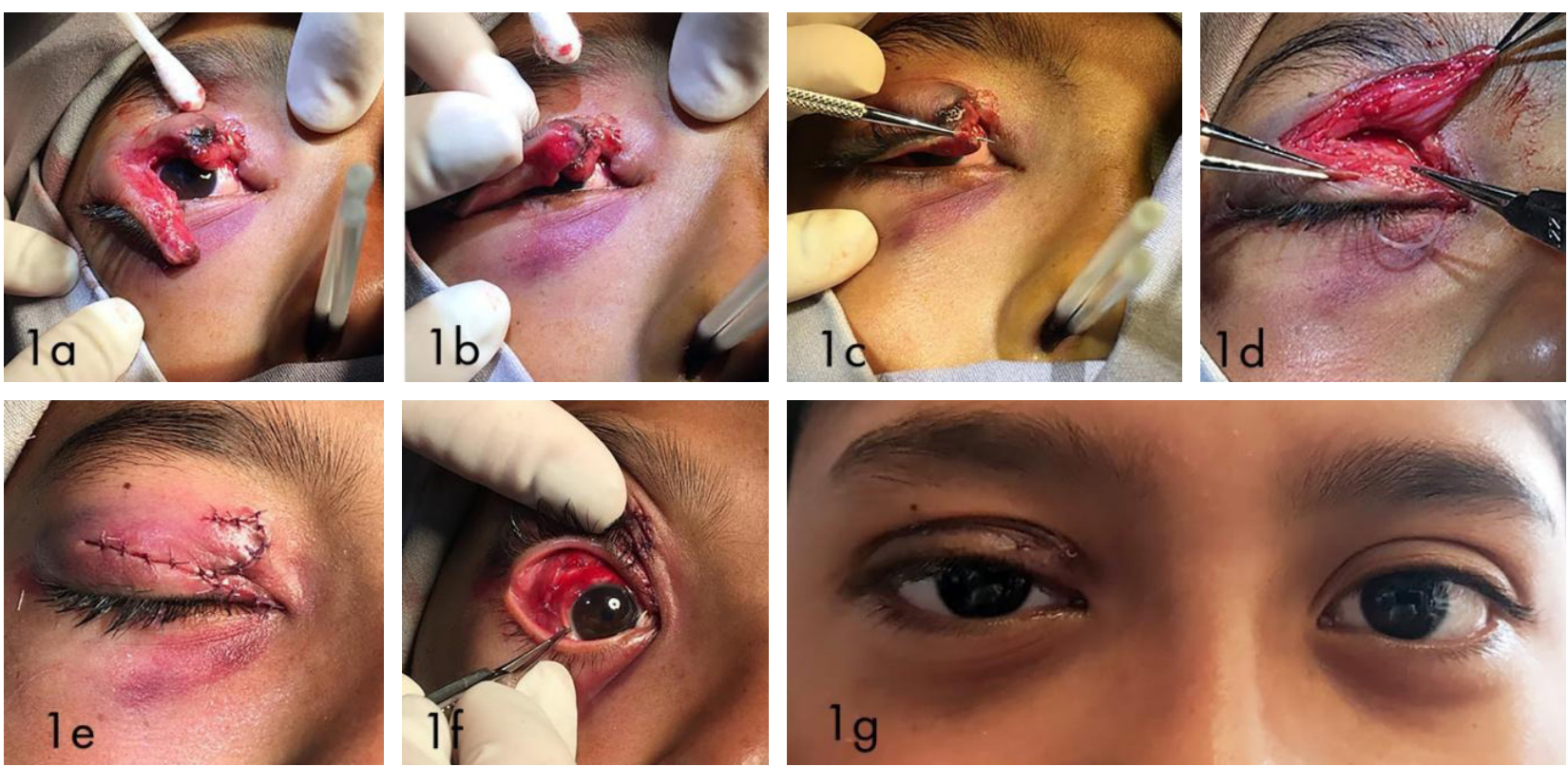

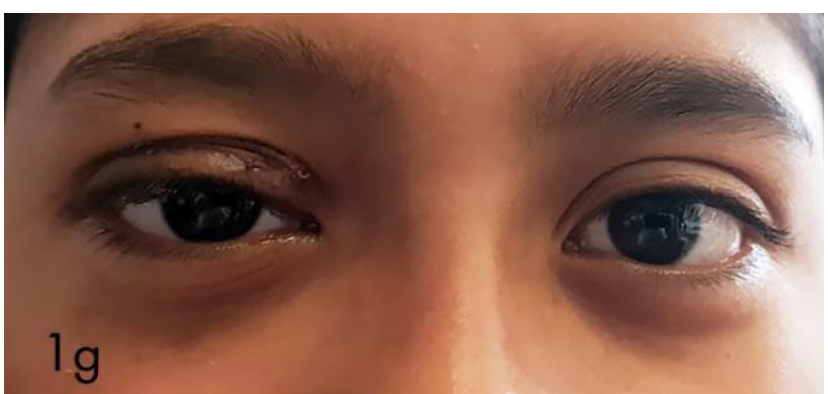

Figure 1a: Right eyelid trauma with upper canaliculus injury. The eyelid is sectioned just above the tarsal plate. Levator aponeurosis is sectioned. Nose is prepped with oxymetazoline $0.05 \%$.

Figure 1b and 1c: The injured canaliculus and distal lumen are identified with surgical loupes and careful dissection with cotton swabs is carried out to identify the lacerated lumen.

Figure 1d: Bicanalicular intubation is made. The stent in position allows a posterior and medial traction permitting an adequate suture of the lacerated tissues.

Figure 1e and 1f: A polyglactin 6-0 suture (Coated Vycryl 910, Ethicon, USA) is used for the repair of all injured eyelid structures including canaliculus and conjunctiva. Levator aponeurosis is also sutured to the tarsus.

Figure 1g: Satisfactory evolution 6 weeks after eyelid and canalicular reconstruction surgery. 
with topical eye drops of $0.3 \%$ tobramycin and $0.1 \%$ dexamethasone (Trazidex, Laboratorios Sophia, Guadalajara, México) and 0.5\% carboxymethylcellulose lubricating eye drops (Refresh Tears, Allergan). Corticosteroid and antibiotic eye drops were tapered and discontinued after 1 month. Patients were examined at days $1,15,30,90$, and then at sixth month for stent removal in the office setting. When needed, skin sutures were removed after 15 days and patients were told not to rub their eyes to prevent stent extrusion.

Anatomical success was considered when the patient reported perception of liquid passing through the nasopharynx when irrigating balanced saline solution through the repaired canaliculus. Epiphora was reported with the Munk score, and functional success was considered when the patient reported being in the first two values, 0-1 (Munk et al, 1990).

To determine the correlation between the presence of epiphora and lack of permeability of injured canaliculus and the presence of epiphora and postoperative complications, a Phi correlation coefficient test was used considering a $\mathrm{p}<0.05$ as statistically significant.

\section{RESULTS}

A total of 10,359 surgical procedures were performed at the Oculoplastic department in the Ophthalmology Institute Conde de Valenciana in Mexico City from 2010 to 2019. Lacrimal canalicular trauma repair corresponded to a total of 208 patients (2\%). All patients were repaired using Crawford bicanalicular stent (Table 1). The most common age of presentation was from 21 to 30 years-old. Patients under the age of 20 corresponded to $45.2 \%$ of the cases. Injury with a sharp object was the most common cause of trauma. The inferior canaliculus was most commonly injured (54.6\%). Results of lacrimal irrigation to the injured canaliculus was performed in 96 patients $(46.2 \%)$. Irrigation was performed after stent removal, even if it was an accidental extrusion prior to 6 months. The data of these 96 patients were included for the correlation analysis of anatomical success. Lacrimal duct permeability (anatomical success) was evaluated in 96 patients and described as permeable in 72 patients $(75 \%)$ and not permeable in 24 patients (25\%). Phi correlation coefficient test showed statistically significant, moderate and directly proportional linear association between the presence of epiphora and the impermeability of the injured canaliculus, ( $\mathrm{r} \varphi$ 0.76, $\mathrm{p}<0.05)$. In 112 patients (53.8\%) lacrimal duct permeability was not reported in the medical records at the $6^{\text {th }}$ month visit. Functional success was reported in 162 patients $(77.8 \%)$ in which Munk scores were found between 0 and 1 . Forty-six patients $(22.2 \%)$ were between $2-4$ values of Munk score. From these, 21 patients underwent superior and inferior canalicular laceration repair. Stent removal was performed in 132 patients at $6^{\text {th }}$ postoperative month. Stent removal prior to 6 months was due to accidental stent extrusion in all cases (76 patients). 
Complications included laceration of the lacrimal punctum, cicatricial ectropion, granuloma formation and creation of false passage, which was seen in $21.4 \%$ of the patients. Phi correlation coefficient test showed a statistically significant, minimal and directly proportional linear association between the presence of epiphora and the presence of complications ( $\mathrm{r} \varphi$ 0.509, $\mathrm{p}<0.05$ ). Most of the patients $(88 \%)$ underwent canalicular injury repair within 72 hours of eye trauma.

Table 1: Clinical characteristics of patients.

\begin{tabular}{|l|c|c|}
\hline Variable & $\mathbf{\%}$ & $(\mathbf{n}=\mathbf{2 0 8})$ \\
\hline GENDER & $\%$ & $(\mathrm{n}=208)$ \\
\hline Male & 80.2 & $(167)$ \\
\hline Female & 19.7 & $(41)$ \\
\hline
\end{tabular}

\begin{tabular}{|l|c|c|}
\hline \hline AGE OF PATIENTS (years) & $(\mathrm{n}=208)$ \\
\hline $0-10$ & 23.1 & $(48)$ \\
\hline 11 to 20 & 22.1 & $(46)$ \\
\hline 21 to 30 & 26.9 & $(56)$ \\
\hline 31 to 40 & 10.1 & $(21)$ \\
\hline 41 to 50 & 10.1 & $(21)$ \\
\hline 51 to 60 & 5.3 & $(11)$ \\
\hline 60 or more & 2.4 & $(05)$ \\
\hline INJURED EYE & $\%$ & $(\mathrm{n}=208)$ \\
\hline Right & 64.9 & $(135)$ \\
\hline Left & 33.7 & $(70)$ \\
\hline Both & 1.4 & $(03)$ \\
\hline
\end{tabular}

OTHER OCULAR INVOLVEMENT. $\mathrm{n}=208$

\begin{tabular}{|l|c|c|}
\hline Yes & 27.4 & $(57)$ \\
\hline No & 72.5 & $(151)$ \\
\hline
\end{tabular}

\begin{tabular}{|l|c|c|}
\hline \multicolumn{2}{|l|}{ MECHANISM OF INJURY } & $\mathrm{n}=208$ \\
\hline Dog bite & 19.7 & $(41)$ \\
\hline Closed Fist & 26.9 & $(56)$ \\
\hline Sharp Object & 33.7 & $(70)$ \\
\hline Fall & 12.5 & $(26)$ \\
\hline Car Accident & 2.9 & $(06)$ \\
\hline Not recorded & 4.3 & $(09)$ \\
\hline
\end{tabular}

TIME OF STENT REMOVAL $\mathrm{n}=208$

\begin{tabular}{|l|c|c|}
\hline$<6$ months & 36.5 & $(76)$ \\
\hline 6 months & 63.4 & $(132)$ \\
\hline
\end{tabular}

\begin{tabular}{|l|c|c|}
\hline \multicolumn{3}{|l|}{ EPIPHORA AT 6 MONTHS. $\mathrm{n}=208$} \\
\hline Yes & 27.4 & $(57)$ \\
\hline No & 72.6 & $(151)$ \\
\hline
\end{tabular}

EPIPHORA SEVERITY *Munk Score. $\mathrm{n}=208$

\begin{tabular}{|l|c|c|}
\hline 0 & 71.6 & $(151)$ \\
\hline 1 & 5.3 & $(11)$ \\
\hline 2 & 11.5 & $(23)$ \\
\hline 3 & 11.1 & $(22)$ \\
\hline 4 & 0.5 & $(01)$ \\
\hline
\end{tabular}

\begin{tabular}{|l|c|c|}
\hline \multicolumn{3}{|l|}{ CANTHAL INVOLVEMENT. $\mathrm{n}=208$} \\
\hline Yes & 37.5 & $(78)$ \\
\hline No & 62.5 & $(130)$ \\
\hline
\end{tabular}

\begin{tabular}{|l|l|l|}
\hline \multicolumn{3}{|l|}{ INVOLVED CANALICULUS. $\mathrm{n}=208$} \\
\hline Superior & 11.1 & $(23)$ \\
\hline Inferior & 54.6 & $(113)$ \\
\hline Both & 34.3 & $(71)$ \\
\hline
\end{tabular}

\begin{tabular}{|l|c|c|}
\hline COMPLICATIONS & $\%$ & $\mathrm{n}=208$ \\
\hline Punctum laceration & 6.2 & $(13)$ \\
\hline Cicatricial ectropion & 8.1 & $(17)$ \\
\hline Granulomas & 6.7 & $(14)$ \\
\hline False Passage & 0.4 & $(01)$ \\
\hline No complications & 78.6 & $(163)$ \\
\hline
\end{tabular}




\begin{tabular}{|l|l|}
\hline Anatomical Success (permeability) / $\mathrm{n}=96$ \\
\hline Yes & $34.6(72) / 75 \%$ \\
\hline No & $11.5(24) / 25 \%$ \\
\hline Not recorded & $53.8(112)$ \\
\hline
\end{tabular}

DURATION OF HOSPITALIZATION (DAYS) n=208

\begin{tabular}{|l|c|c|}
\hline 0 & 59.1 & $(123)$ \\
\hline 1 & 24 & $(50)$ \\
\hline 2 & 9.1 & $(19)$ \\
\hline 4 & 2.9 & $(06)$ \\
\hline$>4$ & 5 & $(01)$ \\
\hline \hline
\end{tabular}

\begin{tabular}{|l|c|c|}
\hline \multicolumn{3}{|l|}{ HOURS FROM INJURY TO SURGERY } \\
\hline$<24$ & 44.7 & $(93)$ \\
\hline $24-48$ & 26.4 & $(55)$ \\
\hline $48-72$ & 17.3 & $(36)$ \\
\hline $72-96$ & 6.7 & $(14)$ \\
\hline$>96$ & 4.8 & $(10)$ \\
\hline
\end{tabular}

\section{DISCUSSION}

A total of 208 patients with nasal bicanalicular intubation with Crawford stent were included in the study $(100 \%)$. At $6^{\text {th }}$ month, functional success was found in $77.8 \%$ and anatomical success was found in $75 \%$. Previous studies report anatomical and functional success ranging between $25 \%$ and $94 \%$ for monocanalicular stents and $40 \%$ to $100 \%$ in bicanalicular stents (Murchinson et al, 2014; Han et al, 2019; Tsai et al, 2018; Kim et al, 2018). Factors contributing to our success range include the retrospective nature of our study, clinical file elaboration by different residents and fellows, surgeries performed by multiple surgeons from 10 different generations, the high premature extrusion rate before and Mexico's City high dry eye prevalence (Murchinson et al, 2014; Martínez et al, 2016). The important highlights that can be remarked from the study are the large patient population and heterogeneous age range, the 10-year duration of the study, the exclusive use of bicanalicular stents and the representative information of a teaching hospital experience.

Most of our epidemiological data and studied variables are comparable to the cited literatures (Jordan et al, 2008; Sendul et al, 2015; Han et al, 2019). The most common age for presentation was in the third decade of life and in male gender, similar to studies by Sendul et al, 2015 and Han et al, 2019. Diffuse injuries were the most common cause of canalicular lacerations, mainly including closed fists, sharp objects (in young adults) and dog bites (in children). The right side and inferior canaliculus were most commonly affected in our study, similar to previous reports and recalling the mechanism of injury explained by Wulc et al (1991). Comparing worldwide, Korea reports up to $25.4 \%$ of bicanalicular lacerations, Taiwan $17.4 \%$ and India 12.5\% (Han et al, 2019). This report is the first study of Mexican population to the knowledge of the authors, and we had a higher percentage of bicanalicular lacerations $(34.3 \%)$. This could be accounted for by the fact that we are a national ophthalmological referral center. Simultaneous globe injury has been reported from 20 to $44 \%$, and in our study, it accounted for $27 \%$ of our patients in accordance with other studies. Most of the patients presented to our hospital the same day or 24 hours later, and at least $70 \%$ were operated within 48 hours. 
The decision to use Crawford stents in a habitual manner resides mainly in the economical factor, and secondarily in the experience we have performing these types of reconstructions. One of the most commented disadvantages of Crawford bicanalicular stents is the potential risk of damage to the uninvolved canaliculus and the technical difficulty of placing the stents. The reports telling about this possible injury to uninvolved canaliculus were first mentioned in 1978, when silicone stents were first being used. We do not consider this to be necessarily certain. We think that a well performed technique does not have to damage an uninvolved canaliculus, and it is also a procedure an oculoplastic surgeon is used to because it is similar to the treatment of congenital nasolacrimal obstruction in children (Tsai et al, 2018). We do not regularly use pigtail probes since we deem this to be a blind procedure that can cause significant damage to the entire lacrimal upper portion, and we prefer direct visualization of the epithelial lining of the lacerated canaliculus. About the technical difficulty of Crawford bicanalicular stents, we agree it can be a more time- taking learning technique, but for a well-trained oculoplastic surgeon it should pose no difficulty, and we believe it provides a better support for the correct suturing of the eyelid, by allowing a posterior and medial traction for a better position of the surgical field. It has even been stated that an accurate layered closure may be functionally more important (Anderson et al, 1979). On the economical side, it is 3 times less expensive to use a Crawford stent than a Monoka Stent. Other mentioned disadvantage is the need for general anesthesia with bicanalicular nasal stents, but we do not consider this a disadvantage since most cases can be well managed by the anesthesiologist and it is easier for our learning fellows to resolve the case adequately if the patient is under general anesthesia. Pediatric patients were always intervened under general anesthesia.

In a more recent review of monocanalicular stents, the complications presented for bicanalicular are "historically" described, such as irritation, damage to the punctum, false passage and granuloma formation (Wladis et al, 2019). It has to be noted that this report is from 1978 when silicon stents were initially being used (Anderson et al, 1979). The most common complication we encountered in our population was cicatricial ectropion, in 17 patients. The second most common complication was punctum slitting or laceration, which we do not believe poses a risk for epiphora and we even consider it can be helpful to prevent it. This happened in 13 patients (6.2\%). Granulomas were found in 14 patients, but this can occur in any surgery where absorbable sutures are used, like the polyglactin we normally use. Complete absorption of polyglactin 910 (Coated Vicryl Ethicon) can take up to 70 days, which can explain why granulomas can occur, and it is not directly associated with the silicon stent. We had no complications of corneal abrasion. The most common complaints from the patients were the nasal extrusion of the stent when sneezing. The 
patients were instructed to put it back in the nose if these occur. We prefer leaving a long stent to retrieve it nasally and avoid inflammation in the reconstructed lacrimal system at the time of the removal.

Another determining factor for the success rates is the time for stent removal. Animal models have shown that 12 weeks are enough for reepithelization, but the exact time for removal is still a matter of debate (Conlon et al, 1994). Nonetheless, it is established that in traumas, at least 6 months of stent permanence is advisable. Fortunately, most of the patients in the study, 132, endured 6 months with the stent. It was quite difficult for pediatric patients to maintain the stent, since they rub their eyes inadvertently. Perhaps another weakness of our study is that the surgeries were mostly performed by oculoplastic fellows, even if most of the times under direct supervision of an oculoplastic faculty surgeon. According to Murchison et al., the degree of training of the surgeon can be accounted for the patient's success prognosis (Murchinson et al, 2014).

There were 46 patients who reported between 2-4 Munk score and continued to report epiphora after 6 months of follow-up. It has been noted that the canaliculus anatomy varies individually, making the site of injury an important factor and having a worse prognosis the closer to the lacrimal punctum (Kennedy et al, 1990; Shi et al, 2012). We didn't consider in our variables the distance from the lacrimal sac to the injury. Anatomical success was verified by tear duct irrigation in 96 patients (46.1\%). From these 96 patients, total permeability of the lacrimal drainage system was found in 72 patients. In our statistical analysis we found that having an obstructed lacrimal canaliculus (lack of anatomical success) or a complication after reconstruction was correlated moderately or minimally directly proportional, respectively. Therefore, it can be asserted that the patient who does not have a patent duct or injured ducts, will have up to a $70 \%$ chance of having an epiphora, and a patient with a surgical complication will have up to a $50 \%$ chance of having epiphora. The presence of granuloma and cicatricial ectropion contribute to the presence of epiphora, assuming that obstruction or lack of permeability of the lacrimal duct may or may not contribute directly to the presence of epiphora. Furthermore, delving into the subjectivity of the epiphora, some patients with an obstructed canaliculus do not manifest epiphora. Factors such as age, gender and ocular surface conditions may be related (Martínez et al, 2016). Furthermore, much discussion has been done in regards to the importance of the inferior canaliculus for tear drainage, being that is the most common canaliculus affected, and still after failed permeability, there are patients that do not refer epiphora, advocating to the equal significance of the superior canaliculus in the tear drainage function and the utmost importance of a correct layered suturing (Reifler 1991; Anderson et al, 1979). 


\section{CONCLUSION}

Crawford bicanalicular stents for monocanalicular or bicanalicular lacerations are a valid and accessible option for canalicular laceration repair. The anatomical and functional success of lacrimal canalicular lacerations repaired with bicanalicular stents in a Mexican teaching hospital is $75 \%$ and $77.8 \%$ respectively. Factors involved in the functional and anatomical success of the reconstruction include presence of postoperative complications and permeability of canaliculus after surgery. The present study displays encouraging results in lacrimal canalicular lacerations repaired with bicanalicular stents in a tertiary referral teaching center in Mexico City.

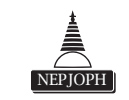

\section{REFERENCES}

Anderson RL, Edwards JJ (1979). Indications, Complications and Results with Silicone Stents. Ophthalmology;86(8):1474-87. doi: 10.1016/s0161-6420(79)35374-x

Conlon MR, Smith KD, Cadera W, Shum D, Allen LH (1994). An animal model studying reconstruction techniques and histopathological changes in repair of canalicular lacerations. Can J Ophthalmol;29(1):3-8. PMid: 8180875

Han S, Wang T, Jia J, Sun S, Fan Y (2019). Bicanalicular Lacerations: Clinical Characteristics and Surgical Outcomes With a New Bicanalicular Silicone Stent. J Craniofac Surg;30(7):2261-4. doi: 10.1097/SCS.0000000000005990

Jordan DR, Ziai S, Gilberg SM, Mawn LA (2008). Pathogenesis of canalicular lacerations. Ophthal Plast Reconstr Surg;24(5):394-8. doi: 10.1097/IOP.0b013e318183267a

Kennedy RH, May J, Dailey J, Flanagan JC (1990). Canalicular laceration. An 11-year epidemiologic and clinical study. Ophthal Plast Reconstr Surg;6(1):46-53. PMid: 2268600

Kersten RC, Kulwin DR (1996). “One-stitch" canalicular repair. A simplified approach for repair of canalicular laceration. Ophthalmology;103(5):785-9. doi: 10.1016/s0161-6420(96)30615-5

Kim YH, Yeo CH, Chung KJ, Lee JH, Kim T (2018). Repair of Lower Canalicular Laceration Using the Mini-Monoka Stent: Primary and Revisional Repairs. J Craniofac Surg;29(4):949-52. doi: 10.1097/SCS.0000000000004423

Liang X, Lin Y, Wang Z, Lin L, Zeng S et al (2012). A modified bicanalicular intubation procedure to repair canalicular lacerations using silicone tubes. Eye;26:1542-7. doi: 10.1038/eye.2012.212

Martinez JD, Galor A, Ramos-Betancourt N, Lisker-Cervantes A, Beltrán F, Ozorno-Zárate J, et al (2016). Frequency and Risk factors associated with dry eye in patients attending a tertiary care ophthalmology center in Mexico City. Clin Ophthalmol;10:1335-42. doi: 10.2147/OPTH.S106451

Munk PL, Lin DT, Morris DC (1990). Epiphora: treatment by means of dacryocystoplasty with balloon dilation of the nasolacrimal drainage apparatus. Radiology;177:687-90. doi: 10.1148/radiology.177.3.2243969

Murchinson AP, Bilyk JR (2014). Canalicular Laceration Repair: An Analysis of Variables Affecting Success. Ophthal Plast Reconstr Surg;30(5):410-4. doi: 10.1097/IOP.0000000000000133 
Naik MN, Kelapure A, Rath S, Honavar S (2008). Management of Canalicular Lacerations: Epidemiological Aspects and Experience with Mini-Monoka Monocanalicular Stent. Am J Ophthalmology;145(2):375-80. doi: 10.1016/j. ajo.2007.09.018

Reifler DM (1991). Management of Canalicular Lacerations. Surv Ophthalmol;36(2): 113-32. doi: 10.1016/00396257(91)90125-y

Sendul SY, Cagatay HH, Dirim B, Demir M, Cinar S, Ucgul C, et al (2015). Reconstructions of Traumatic Lacrimal Canalicular Lacerations: A 5 Years Experience. Open Access J Sci Technol;3:1-6. doi: 10.11131/2015/101121

Shi W, Fan X (2012). Incidence of Postoperative Obstruction at Different Sites of Lacrimal Canalicular Injury. Plast Reconstr Surg;29(4):731e-733e. doi: 10.1097/PRS.0b013e318245e779

Tint NL, Alexander P, Cook AE, Leatherbarrow B (2011). Eyelid avulsion repair with bi-canalicular silicone stenting without medial canthal tendon reconstruction. Brit J Ophthalmol;95(10):1389-92. doi: 10.1136/bjo.2010.196246

Tsai MJ, Lin IW, Lee SS, Lai CS (2018). Stitch guide technique for bicanalicular nasal intubation in single canalicular laceration reconstruction. Kaohsiung J Med Sci; 34:529-34. doi: 10.1016/j.kjms.2018.04.007

WladisEJ,AakaluVK, TaoJP, etal(2019).MonocanalicularStentsinEyelidLacerations. Ophthalmology;126(9):1324-9. doi: 10.1016/j.ophtha.2019.03.045

Wu SY, Ma L, Chen RJ, Tsai YJ, Chi YC (2010). Analysis of Bicanalicular Nasal Intubation in the Repair of Canalicular Lacerations. Jpn J Ophthalmol;54:24-3. doi: 10.1007/s10384-009-0755-7

Wulc AE, Arterberry JF (1991). The Pathogenesis of Canalicular Lacerations. Opthalmology;98(8):1243-9. doi: 10.1016/s0161-6420(91)32148-1 